\title{
A systematic review of the risk factors for suicidal ideation, suicidal attempt and completed suicide among children and adolescents in sub-Saharan Africa between 1986 and 2018: protocol for a systematic review of observational studies
}

Godfrey Zari Rukundo ${ }^{1 *}$ (D) Elizabeth Kemigisha ${ }^{2}$, Moses Ocan ${ }^{3}$, Wilson Adriko ${ }^{4}$ and Dickens Howard Akena ${ }^{5}$

\begin{abstract}
Background: Suicide is one of the leading causes of death among children and adolescents. Most studies about the burden and risk factors for suicide have been conducted in high-income countries. However, there is a dearth in the literature about the burden and risk factors for suicide among children and adolescents in low- and middle-income countries including within Sub-Saharan Africa (SSA). There is need to summarise the available literature about the burden and risk factors for suicide among children and adolescents in SSA. In this review, we will (a) determine the overall prevalence of suicidal ideation, suicidal attempt and completed suicide among children and adolescents in SSA; (b) describe the methods (such as hanging, firearms, overdose, poisoning, drowning and burning) used for suicidal attempt, and completed suicide among children and adolescents in SSA; and (c) document the risk factors for suicidal ideation, suicidal attempt and completed suicide among children and adolescents in SSA.

Methods: The review will be conducted and reported in accordance to the preferred reporting items for systematic reviews and meta-analysis (PRISMA) statement. We will include journal articles that have documented the prevalence and risk factors for suicidal ideation, suicidal attempt and completed suicide among children and adolescents aged 5-19 years in SSA. We will also include accessible grey literature about the topic. Qualitative studies will be excluded from the study since they are limited in estimating prevalence. We will search different search engines including PUBMED, EMBASE, Psych-INFO, Cochrane Library, Africa wide-information and global health using suicide, adolescents and children, SSA as the keywords. We will use a meta-analysis, should we find that there is no heterogeneity between included studies.

Discussion: This protocol describes a systematic review of observational studies reporting completed suicide, suicidal ideation and suicidal attempt among children and adolescents in sub-Saharan Africa. We anticipate that once this review is complete and published, our findings will be of interest to adolescents with suicidal behaviour, their families and caregivers, clinicians and other healthcare professionals, scientists and policy makers.
\end{abstract}

Systematic review registration: PROSPERO International prospective register of systematic reviews: CRD42016048610. Keywords: Suicide, Suicidal attempt, Suicidal ideation, Suicidal behaviour, Adolescents

\footnotetext{
* Correspondence: grukundo@must.ac.ug

${ }^{1}$ Department of Psychiatry, Mbarara University of Science and Technology

and African Centre for Suicide Research, Mbarara, Uganda

Full list of author information is available at the end of the article
}

(c) The Author(s). 2018 Open Access This article is distributed under the terms of the Creative Commons Attribution 4.0 International License (http://creativecommons.org/licenses/by/4.0/), which permits unrestricted use, distribution, and reproduction in any medium, provided you give appropriate credit to the original author(s) and the source, provide a link to the Creative Commons license, and indicate if changes were made. The Creative Commons Public Domain Dedication waiver (http://creativecommons.org/publicdomain/zero/1.0/) applies to the data made available in this article, unless otherwise stated. 


\section{Background}

Globally, suicide is a public health concern and one of the leading causes of death among children and adolescents [1]. Most studies about the burden and risk factors for suicide have been conducted in high-income countries (HIC). However, there is a dearth in the literature about the burden and risk factors for suicide among children and adolescents in low- and middle-income countries (LMIC) including within Sub-Saharan Africa (SSA); the majority of studies about the risk factors for suicide having been conducted among adults and in specific sub-populations [2-5]. A recent literature review by Mars et al. (2014) documented the prevalence of suicide and its risk factors on the African continent, but fell short of studying children and adolescents $[6,7]$. The African annual prevalence of suicide was found to be 34,000 with incidence of 3.2 per 100,000 .

Suicide is the third leading cause of death among adolescents and young adults [8-10]. A number of factors including high levels of poverty, growing unemployment, disruptions in traditional family life and the unrecognised and poorly treated depressive illness, political instabilities and high disease burdens including HIV/AIDS disproportionately affect children and adolescents in SSA $[6,11]$, and places them at a much higher risk of suicide, especially in those who fail to cope with these upheavals [12]. Moreover, SSA is home to the highest population of children and adolescents, with $20 \%$ of the world's children and adolescents living in SSA. In South Africa, suicidality has been reported at suicide attempt $3.2 \%$, suicide planning $5.8 \%$ and suicidal ideation $7.2 \%$ [11]. The few studies that have documented the burden and risk factors of suicide among children and adolescents in SSA have reported conflicting results, perhaps as a result of differences in the methods of assessment [13].

There is an urgent need to summarise the available literature about the burden and risk factors for suicide among children and adolescents in SSA. Findings from the synthesis of this data will be useful in developing specific evidence-based prevention and treatment strategies. Findings from this review will also be used in enlightening parents, children, adolescents and members of the community at large about the burden of suicide and the factors that predispose individuals to commit suicide.

For this review, we will (a) determine the overall prevalence of suicidal ideation, suicidal attempt and completed suicide among children and adolescents in SSA; (b) describe the methods used for suicidal attempt and completed suicide among children and adolescents in SSA; and (c) document the risk factors for suicidal ideation, suicidal attempt and completed suicide among children and adolescents in SSA.

\section{Methods}

The review will be conducted and reported in accordance to the preferred reporting items for systematic reviews and meta-analysis (PRISMA) statement (Additional file 1) $[14,15]$. This systematic review protocol title has been registered with the International Prospective Register of Systematic Reviews (PROSPERO) database (registration number: CRD42016048610).

\section{Eligibility criteria}

We will include articles that have documented the prevalence and risk factors for suicidal ideation, suicidal attempt and completed suicide among children and adolescents aged 5-19 years in SSA. The review will include articles from studies done following cross-sectional, longitudinal, cohort and case control study designs. Sub-group analysis will be performed on the included studies to cater for the variations in the study designs.

Unpublished but accessible studies will be included in the review. Studies on non-suicidal deliberate self-harm will be excluded. Articles with only abstracts available (with no full articles) will be excluded as they may be difficult to compare with full articles.

The selection criteria of the articles for inclusion will be strictly applied and no double reporting of the same outcome will be included in the review. We will report the study as a single one, but in the event that there were separate outcomes, we will report them as such. Each publication will have the name of the author, year and labelled a, b, c, d.

\section{Study design}

We will conduct a systematic review of observational studies (cross-sectional, cohort, longitudinal and case control studies) that documented the prevalence of completed suicide, suicide attempt or suicidal ideation among children and adolescents living in SSA irrespective of how the studies report the main outcome. We will also review suicide methods used and associated risk factors for suicide.

\section{Setting}

The systematic review will only include studies conducted in sub-Saharan Africa.

\section{Participants}

The participants in this systematic review will be children and adolescents aged 5-19 years living in SSA.

\section{Comparisons}

The comparison groups will be children and adolescents without suicidal ideation, suicidal attempt and completed suicide. 


\section{Outcomes}

The outcomes of interest in this systematic review will be [1] prevalence, [2], suicidal methods used and [3] risk factors for suicidal ideation, attempts and completion among children and adolescents in Sub-Saharan Africa.

\section{Search methods}

The search strategy will be carried out by the research team using the following electronic databases and search engines, from inception, using the same search strategy with alterations as appropriate for each database: the Cochrane Library, PsychINFO, PubMed, EMBASE, Africa wide-information and global health. We will hand search the references of the included studies. We will develop a Medline search strategy and use a combination of mesh terms, text words and combine them with appropriate Boolen operators in order to identify as many studies as possible. Grey literature will also been included. We will identify the relevant grey literature using web searching, web-based catalogues as well as using bibliographic databases.

\section{Search string}

We will search using various terms as shown in Appendix.

\section{Data extraction}

The summary of the variables from which data will be extracted from reviewed articles include citation of the study, country/region where the study was done, year when data collection was carried out, study design, sample size, response rate, method of data collection, study population, age of study participants, selection criteria of the study participants, gender, religion, ethnicity, prevalence of suicidal ideation, prevalence of attempted suicide, prevalence of completed suicide, method of data analysis and sampling criteria used in participant selection. Quality of the study will be assessed through risk of bias assessment tool which will be done using a checklist developed from the strobe statement [16].

The data collected will also include the authors' names, the title of the article and the year the study was conducted. This data will be extracted by two content experts on the research team. For articles with missing information, the primary authors will be contacted by the principle investigator to provide the missing or additional data. For any discrepancies, the principle investigator will make the final decision.

Data extraction will be done in two stages. Mr. Wilson Adriko, an information scientist and Dr. Elizabeth Kemigisha, a Paediatrician with interest in adolescent health will screen the title and abstract of all identified studies. We will then download the full text articles for further screening by two independent reviewers: Dr. Moses Ocan, a Pharmacologist with expertise in conducting reviews and Dr. Dickens H. Akena, a Psychiatrist and systematic review specialist. In the event that there is disagreement about which study to include or exclude, the PI who is a content expert will be the arbitrator.

\section{Quality assessment}

Data will be entered in REVMAN 5.1.2 software for analysis of findings. The assessment of the methodological quality of the articles will be done using QUIPS (Quality in Prognostic Studies). We will assess study participation, confounding measurement and handling, outcome measurement and statistical analysis, and presentation.

The quality of primary studies will be ensured as part of the selection criteria of the articles prior to inclusion into the systematic review. This will be done by ensuring that only studies that meet the a priori selection criteria will be included in the review [17]. Screening of articles for inclusion into the review will be done independently by two reviewers. Articles will only be included if the Kappa agreement between the two reviewers in $\geq 70 \%$. In addition, risk of bias (publication bias, methodological bias) in the included articles will be assessed using a risk of bias assessment checklist developed from the Cochrane risk of bias (RoB) assessment tool. We will use the AMSTAR [18] guidelines to assess for the quality of included studies, and GRADE [19] to assess the overall quality of each of the outcomes.

\section{Data synthesis}

\section{Qualitative synthesis}

We will describe the important study features like date, number of participants, age categories, prevalence, completed suicide, attempted suicide, suicidal ideation, country in which the study was done and the associated risk factors. We will export the data to STATA 13.1 for analysis.

\section{Meta-analysis}

Statistical tests for heterogeneity will be used to assess the degree of variability in the prevalence measures between the included studies. Specifically, we will use the $I^{2}$ statistic to report the percentage of variation across studies that is due to heterogeneity [20]. This is preferred because it does not depend on the number of studies reviewed. The $I^{2}$ score of 0 (zero) will be considered no heterogeneity, whereas a score of more than zero but less than $25 \%$ will be taken as low heterogeneity. On the other hand, an $I^{2}$ score of more than $25 \%$ will be considered as moderatehigh heterogeneity. In case we find no statistically significant heterogeneity ( $I^{2}=$ zero), we will go ahead and conduct meta-analysis. Otherwise, in circumstances of statistically significant heterogeneity, we will not conduct meta-analysis [21]. 
Random-effects models will be employed [22]. This model is used when the researcher thinks or knows that the effect/main outcome varies widely in the population. We will use random effects model, because we are not confident that all the variables we will identify will be measured in the same way and with the same values [23-25].

The prevalence, odds ratios and confidence intervals of individual studies will be presented in forest plots and we will generate a summary prevalence and confidence levels. There will also be sensitivity and sub-group analyses to determine the influence of selected independent variables on the effect size (suicidality). We will use funnel plots to show small-study effects that will help point towards publication bias.

In addition to the prevalence, we will also pool effect estimates relating to risk factors. This will be achieved through the random effects meta-analysis. Pooling of results will be done for the prevalence of suicidal ideation, attempt and completed suicide. This will only be done if there is need to proceed to meta-analysis. We will conduct the following sub-group analyses: study designs (cross sectional studies, case control studies, clinical trials and cohort studies), region (s) where the studies were conducted, gender and participant age. This is to ensure that only studies that report similar effect measures are compared. In the event that there is too much heterogeneity, we will summarise the studies as narrative reviews.

The included articles will report study variables of interest to the review differently. The interconversion of the measurements of interest will therefore depend on how the articles have reported and available data to that effect. However, the interconversion will be manually done whenever there is need. The review will use odds ratios (OR) in reporting the risk factors for suicidal ideation, attempted suicide and completed suicide. Using Stata 13, we shall perform meta-regression on articles with high heterogeneity. The subgroup and meta-regression analyses for levels of heterogeneity will be conducted since heterogeneity may often be missed or undetected in the included studies. The possible causes of variability that will need to be considered include variation in participants from the different settings, outcomes of the different studies (suicidal ideation, attempt, behaviour), varying degrees of bias in the different studies and varying power of the different studies.

Heterogeneity will be inferred from the $I^{2}$-statistic $\left(I^{2}=25 \%\right.$ (small heterogeneity), $I^{2}=50 \%$ (moderate heterogeneity), $I^{2}=75 \%$ (large heterogeneity)) generated using Stata 13 software [26].

\section{Discussion}

This protocol describes a systematic review of observational studies reporting completed suicide, suicide attempt and suicidal ideation among children adolescent in sub-Saharan Africa. According to our knowledge, no previous systematic review specifically addressed this topic. We will summarise the methods used and results of observational studies specifically looking at the prevalence and risk factors for completed suicide and suicidal behaviour in Sub-Saharan Africa. We anticipate facing a challenge of varying methodologies and use of different terms to mean the same thing. We also anticipate the challenge of stigma and underreporting of suicide-related deaths or morbidity. In addition, studies may have been conducted in various study populations like adolescents with HIV, or in war torn areas, those in school, with few studies looking at the general population. There may also be unpublished studies conducted on the same subject in the region that we may not have access to, but we shall include any available grey literature. We anticipate that once this review is complete and published, our findings will be of interest to adolescents with suicidal behaviour, their families and caregivers, clinicians and other healthcare professionals, scientists and policy makers.

\section{Appendix}

\section{Search string}

We will search using the following terms in the order 1-3.

1. Suicide) OR Para-suicide) OR suicide awareness) OR suicide attempt) OR attempted suicide) OR suicide ideation) OR potential suicide) OR deliberate self-harm) OR uncompleted suicide) OR completed suicide) OR suicidality

2. Children) OR child) OR boy) OR girl) OR juvenile) OR minors) OR paediatric) OR Adolescence) OR Preadolescence) OR Puberty) OR Teenager) OR Teen) OR Young) OR Youth

3. Developing countries) OR low income countries) OR resource-limited) OR resource constrained) OR Africa) OR Angola) OR Benin) OR Botswana) OR Burkina Faso) OR Burundi) OR Cameroon) OR Cape Verde) OR Central African Republic) OR Chad) OR Comoros) OR Congo) OR Democratic Republic of Congo) OR Djibouti) OR Equatorial Guinea) OR Eritrea) OR Ethiopia) OR Gabon) OR Gambia) OR Ghana) OR Guinea) OR Guinea Bissau) OR Ivory Coast) OR Cote d'Ivoire) OR Jamahiriya) OR Jamahiryia) OR Kenya) OR Lesotho) OR Liberia) OR Madagascar) OR Malawi) OR Mali) OR Mauritania) OR Mauritius) OR Mayote) OR Mozambique) OR Mozambique) OR Namibia) OR Niger) OR Nigeria) OR Principe) OR Reunion) OR Rwanda) OR Sao Tome) OR Senegal) OR Seychelles) OR Sierra Leone) OR Somalia) OR South Africa) OR St Helena) OR Sudan) OR Swaziland) OR Tanzania) OR Togo) OR Tunisia) 
OR Uganda) OR Western Sahara) OR South Suda) OR Zambia) OR Zimbabwe 657,258

\section{Additional file}

Additional file 1: PRISMA-P 2015 Checklist. (DOCX $81 \mathrm{~kb})$

\section{Abbreviations}

SA: Suicide attempt; SI: Suicide ideation; WHO: World Health Organisation

\section{Acknowledgements}

We would like to thank Professor Celestino Obua for supporting this review by providing training on how to conduct systematic reviews through his grant "Mbarara" University Research Training Initiative (MURTI) Program (Grant Number D43TW010128) funded by Fogarty International Center of the National Institutes of Health.

\section{Funding}

There is no dedicated funding for this review.

\section{Availability of data and materials}

Not applicable.

\section{Authors' contributions}

Content expert: GZR, a Child and Adolescent Psychiatrist with a PhD in suicidology. Search strategist: WA. Data abstraction and extraction and entry: EK, a Paediatrician, and MO, a Pharmacologist. DHA a Psychiatrist and systematic review specialist based at the African Centre for Systematic Reviews. All authors read and approved the final manuscript.

\section{Authors' information}

Dr. G. Z. Rukundo is the content expert. He is a Child and Adolescent Psychiatrist with a PhD in suicidology.

Mr. Wilson Adriko is a Librarian and search strategist.

Dr. Elizabeth Kemigisha, a Paediatrician, and Dr. Moses Ocan, a

Pharmacologist will do data abstraction, extraction and entry.

Dr. Dickens Akena, a Psychiatrist with PhD in systematic reviews and is based at the African Centre for Systematic Reviews

\section{Ethics approval and consent to participate}

No ethical approval will be required for the performance of this systematic review.

\section{Consent for publication}

No need for consent for publication. No raw data will be used. We will use already published data.

\section{Competing interests}

The authors declare that they have no competing interests.

\section{Publisher's Note}

Springer Nature remains neutral with regard to jurisdictional claims in published maps and institutional affiliations.

\section{Author details}

${ }^{1}$ Department of Psychiatry, Mbarara University of Science and Technology and African Centre for Suicide Research, Mbarara, Uganda. ${ }^{2}$ Faculty of Interdiscilinary Studies, Mbarara University of Science and Technology, Mbarara, Uganda. ${ }^{3}$ Department of Pharmacology \& Therapeutics, Makerere College of Health Sciences, Kampala, Uganda. ${ }^{4}$ Library, Mbarara University of Science and Technology, Mbarara, Uganda. ${ }^{5}$ Department of Psychiatry, Makerere College of Health Sciences and African Centre for Systematic, Kampala, Uganda.
Received: 5 October 2017 Accepted: 28 November 2018

Published online: 12 December 2018

\section{References}

1. Wasserman D, Cheng $Q$, Jiang GX. Global suicide rates among young people aged 15-19. World Psychiatry. 2005;4(2):114-20

2. Rukundo GZ, Mishara BL, Kinyanda E. Burden of suicidal ideation and attempt among persons living with HIV and AIDS in Semiurban Uganda. AIDS Res Treat. 2016;2016:3015468.

3. Kinyanda $E$, Weiss HA, Mungherera M, Onyango-Mangen $P$, Ngabirano E, Kajungu $\mathrm{R}$, et al. Prevalence and risk factors of attempted suicide in adult war-affected population of eastern Uganda. Crisis. 2013;34(5):314-23.

4. Kinyanda E, Hoskins S, Nakku J, Nawaz S, Patel V. The prevalence and characteristics of suicidality in HIV/AIDS as seen in an African population in Entebbe district, Uganda. BMC Psychiatry. 2012;12:63.

5. Rukundo GZ, Mishara B, Kinyanda E. Psychological correlates of suicidality in HIV/AIDS in semi-urban South-Western Uganda. Trop Dr. 2016. https://doi. org/10.1177/0049475515623110.

6. Mars B, Burrows S, Hjelmeland H, Gunnell D. Suicidal behaviour across the African continent: a review of the literature. BMC Public Health. 2014;14:606

7. Mars B, Heron J, Crane C, Hawton K, Kidger J, Lewis G, et al. Differences in risk factors for self-harm with and without suicidal intent: findings from the ALSPAC cohort. J Affect Disord. 2014;168:407-14

8. Zhao S, Zhang J. Suicide risks among adolescents and young adults in rural China. Int J Environ Res Public Health. 2014;12(1):131-45.

9. Borowsky IW, Resnick MD, Ireland M, Blum RW. Suicide attempts among American Indian and Alaska native youth: risk and protective factors. Arch Pediatrics Adolesc Med. 1999;153(6):573-80

10. Suicide is a Leading Cause of Death in the United States [Internet]. 2015. Available from: https://uk.search.yahoo.com/search?fr=mcafee\&type= C211GB714D20180309\&p=suicide+is+a+leading+cause+of+death+in+the +US. Accessed 7 Dec 2018.

11. Cluver L, Orkin M, Boyes ME, Sherr L. Child and adolescent suicide attempts, suicidal behavior, and adverse childhood experiences in South Africa: a prospective study. J Adolesc Health. 2015:57(1):52-9.

12. Randall JR, Doku D, Wilson ML, Peltzer K. Suicidal behaviour and related risk factors among school-aged youth in the Republic of Benin. PLoS One. 2014; 9(2):e88233

13. Page A, Lewis G, Kidger J, Heron J, Chittleborough C, Evans J, et al. Parental socio-economic position during childhood as a determinant of self-harm in adolescence. Soc Psychiatry Psychiatr Epidemiol. 2014:49(2):193-203.

14. Moher D, Shamseer L, Clarke M, Ghersi D, Liberati A, Petticrew M, et al. Preferred reporting items for systematic review and meta-analysis protocols (PRISMA-P) 2015 statement. Syst Rev. 2015;4:1

15. Moher D, Liberati A, Tetzlaff J, Altman DG, Group P. Preferred reporting items for systematic reviews and meta-analyses: the PRISMA statement. Open Med. 2009;3(3):e123-30.

16. Vandenbroucke JP, von Elm E, Altman DG, Gotzsche PC, Mulrow CD, Pocock $\mathrm{SJ}$, et al. Strengthening the reporting of observational studies in epidemiology (STROBE): explanation and elaboration. Ann Intern Med. 2007; 147(8):W163-94

17. Ocan M, Obuku EA, Bwanga F, Akena D, Richard S, Ogwal-Okeng J, et al. Household antimicrobial self-medication: a systematic review and meta-analysis of the burden, risk factors and outcomes in developing countries. BMC Public Health. 2015;15:742.

18. Sequeira-Byron P, Fedorowicz Z, Jagannath VA, Sharif MO. An AMSTAR assessment of the methodological quality of systematic reviews of oral healthcare interventions published in the journal of applied Oral science (JAOS). J Appl Oral Sci. 2011;19(5):440-7.

19. Meader N, King K, Llewellyn A, Norman G, Brown J, Rodgers M, et al. A checklist designed to aid consistency and reproducibility of GRADE assessments: development and pilot validation. Syst Rev. 2014;3:82.

20. Ma X, Lin L, Qu Z, Zhu M, Chu H. Performance of between-study heterogeneity measures in the Cochrane library. Epidemiology. 2018;29(6):821-4.

21. Kontopantelis E, Springate DA, Reeves D. A re-analysis of the Cochrane library data: the dangers of unobserved heterogeneity in meta-analyses. PLOS One. 2013:8(7):e69930.

22. Demidenko E, Sargent J, Onega T. Random effects coefficient of determination for mixed and meta-analysis models. Commun Stat Theory Methods. 2012;41(6):953-69. 
23. Bown MJ, Sutton AJ. Quality control in systematic reviews and metaanalyses. Eur J Vasc Endovasc Surg. 2010;40(5):669-77.

24. O'Rourke K, Detsky AS. Meta-analysis in medical research: strong encouragement for higher quality in individual research efforts. J Clin Epidemiol. 1989;42(10):1021-4.

25. Sally HJPaG, editor. Cochrane handbook for systematic reviews of Interventions. 2011.

26. Harrod CS, Goss CW, Stallones L, DiGuiseppi C. Interventions for primary prevention of suicide in university and other post-secondary educational settings. Cochrane Database Syst Rev [Internet]. [cited 2018 Feb 28];(10). Art. No.: CD009439. https://doi.org/10.1002/14651858.CD009439.pub2. Available from: http://onlinelibrary.wiley.com/doi/10.1002/14651858.CD009439.pub2/full.

Ready to submit your research? Choose BMC and benefit from:

- fast, convenient online submission

- thorough peer review by experienced researchers in your field

- rapid publication on acceptance

- support for research data, including large and complex data types

- gold Open Access which fosters wider collaboration and increased citations

- maximum visibility for your research: over $100 \mathrm{M}$ website views per year

At BMC, research is always in progress.

Learn more biomedcentral.com/submissions 